\title{
Investigating Energization Transients and the Potentiality of Partial Discharge Inception and Damage in Nanofilled Polypropylene Insulation for DC Cables and Capacitors
}

\author{
P. Seri \\ DEI \\ University of Bologna \\ Bologna, Italy \\ H. Naderiallaf \\ $D E I$ \\ University of Bologna \\ Bologna, Italy
}

\author{
R. Ghosh \\ DEI \\ University of Bologna \\ Bologna, Italy \\ G.C. Montanari \\ Center for Advanced Power Systems \\ Florida State University \\ Tallahasse, USA
}

\begin{abstract}
New nanostructured insulating materials are investigated, within the European project GRIDABLE, to be used for DC cable and capacitors. Besides electrical, thermal and mechanical properties, and life behavior, work is being done to evaluate their capability to endure highly stressing conditions as those cause by the inception of partial discharges, PD. This paper, in particular, analyzes what happens during energization of a DC cable or capacitors, when voltage goes from zero to the nominal value in a few seconds, while the internal electrical field takes longer time to reach the DC steady state configuration. During an energization transient, indeed, electrical field in insulation, and insulation defects (as cavities), is driven by permittivity, not conductivity as in steady state. Hence, PD might occur with high repetition rate, which would not occur, or at much lower repetition rate, in steady state. The way to evaluate the time constant of the transient, that is, through charging current measurement, is described and successfully fitted to the results of PD measurements performed on two types of polypropylene, PP: neat and nanostructured, and a cross-linked polyethylene, XLPE, having significantly different electrical characteristics.
\end{abstract}

Keywords-nanodielectrics, polypropylene, HVDC, partial discharges

\section{INTRODUCTION}

A new European project, that is, GRIDABLE (Polymer Nanocomposite Insulation Material Enabling Integration of Renewables and DC Storage Technologies in the AC Energy Grid) was launched under the HORIZON 2020 framework funded by the European Community. The aim of the project is to develop a new generation of nanostructured polymeric materials suitable for DC cables and capacitors, based on thermoplastic, i.e. polypropylene (PP), polymers and silica, $\mathrm{SiO} 2$, as nanofiller. The main objective is to improve the electrical and thermal properties and reach higher design field and temperature for the same design life and reliability of the present DC technology, that is, XLPE (cross-linked polyethylene) for cables and PP for capacitors.

Significant work has been done already dealing with steady state DC field, from conductivity to space charge and breakdown voltage measurements [1-5], as well as on the partial discharge inception voltage $[6,7]$.
However, both capacitors and cables under DC will experience during life several energization transients, and most likely voltage polarity inversions, [8], during which the electric field distribution inside insulation and insulation defects (as cavities) will not be driven by conductivity, as in DC steady state, but, at least at time $0+$, by permittivity as in AC. This might cause unexpected highly-damaging phenomena as partial discharges to occur during transients, and, thus, cause insulation accelerated aging and early breakdown. It is noteworthy that the transient regime regarding electric field in DC insulation does not coincide with the very short one relevant to the establishment of the supply voltage on the object under test, which is associated with generator and load characteristics and, in general, may take seconds. The latter is that considered in any type of e.g. MV to HV cable test (e.g. [9]), the former is often not mentioned, not known and certainly not standardized.

This paper has the purpose to investigate such phenomena and how nanostructuration of a base polymer, having already very good characteristics, as PP, can help in handling electric field transients and their potential harmfulness.

\section{ELECTRIC FIELD DURING ENERGIZATION TRANSIENT}

While the transient to establish the test or operating voltage on a DC apparatus is fast, ranging from seconds to minutes, the time needed to reach steady-state field distribution, driven purely by conductivity, in insulation is much longer. The equations governing the electric field transient are e.g. shown in $[10,11]$. Summarizing, the transient time to reach steady DC can be approximately expressed by:

$$
\tau \propto \frac{\varepsilon_{e q}}{\sigma_{T}}
$$

where $\sigma_{T}$ is conductivity and $\varepsilon_{e q}$ the equivalent permittivity as derived from the time constant obtained from polarization current measurements performed on the insulating material used for the apparatus, at the chosen steady-state DC field and temperature, see [12]. 
As an example, Fig. 1 shows the voltage energization and the electric transients (from the set of relevant Maxwell equations $[10,11])$ to reach DC steady state for a uniform field configuration test object (multi-layer films), considering an energization ramp with a duration of $30 \mathrm{~s}, \sigma_{T}=3 \cdot 10^{(-16)}$ $\mathrm{S} / \mathrm{m}, \varepsilon_{e q}=3, \tau=88600 \mathrm{~s}$. Figure 1 reports also the electric field in a cavity during the transient to steady state, together with the theoretical PD inception field, derived from the approximate Niemeyer expression [13]. Figure 2 shows an electric field simulation in a MV XLPE cable with a spherical internal defect filled with air under (a) AC and (b, c) DC power supply. Figure 2 (b) is obtained when the inner conductor is at $45^{\circ} \mathrm{C}$, while Fig. 2(c) when the inner conductor is at $90^{\circ} \mathrm{C}$, thus under significantly different values of polymer conductivity, being the outer conductor temperature fixed at $35^{\circ} \mathrm{C}$.

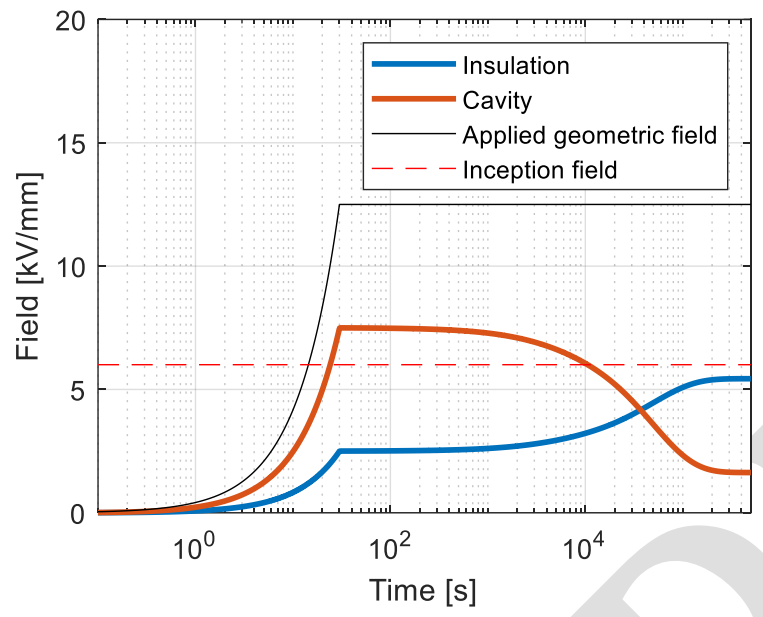

Fig. 1. Electric field in a cavity embedded in a test object (parallel electrodes) during transient to DC steady state (under the hypothesis of no $\mathrm{PD}$ activity), together with the theoretical PD inception field, derived from the approximate Niemeyer expression [13].

Two fundamental considerations can be drawn from these figures. One is that the electric field in a cavity can vary drastically from $\mathrm{AC}$ to $\mathrm{DC}$, depending on permittivity ratio for AC, and on conductivity ratio in DC, see Figs. 2(a) and (b). The other is that any inference regarding electrical field behavior in DC steady state must account for insulation conductivity, thus temperature, and hence on apparatus loading, while this holds much less in AC. This is due to the fact that the exponential dependence of conductivity on temperature is much more significant than that of permittivity and, on the other hand, while conductivity in a polymer can increase of orders of magnitude when temperature rises, that of the gas (generally air) filling a cavity does not, see Figs. 2(b) and (c). This means that the partial discharge inception voltage in DC steady state can be both significantly larger than in AC (thus than the value that shall be considered during the energization transient), and lower, depending on the conductivity of the insulation, and its temperature coefficient, $\alpha$. Indeed, the simplified expression:

$$
\sigma(T)=\sigma_{0} \exp (\alpha \mathrm{T})
$$

where $T$ is temperature in ${ }^{\circ} \mathrm{C}, \alpha$ is the temperature coefficient and $\sigma_{0}$ is the reference conductivity (at $0{ }^{\circ} \mathrm{C}$ ), can be used for the conductivity dependence on temperature. According to (2) and the inception field relationship in [7, 8, 13], the ratio PDIV $_{\mathrm{AC}} / \mathrm{PDIV}_{\mathrm{DC}}$, according also to [6], can go e.g. from 1.75 to 0.5 for $\sigma_{0}=10^{-15} \mathrm{~S} / \mathrm{m}, \alpha=0.04{ }^{\circ} \mathrm{C}^{-1}$ and a temperature from $20^{\circ} \mathrm{C}$ to $90^{\circ} \mathrm{C}$ (for a multi-layer specimen with an artificial cavity in the middle $0.1 \mathrm{~mm}$ thick where PD can incept).

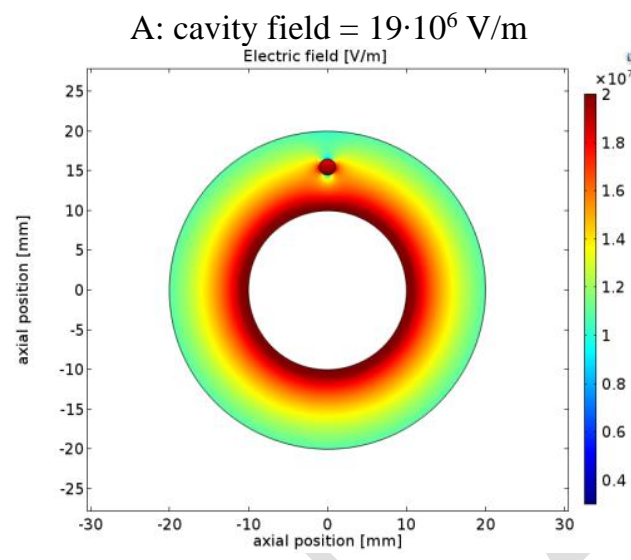

(a)

B: cavity field $=2.3 \cdot 10^{6} \mathrm{~V} / \mathrm{m}$

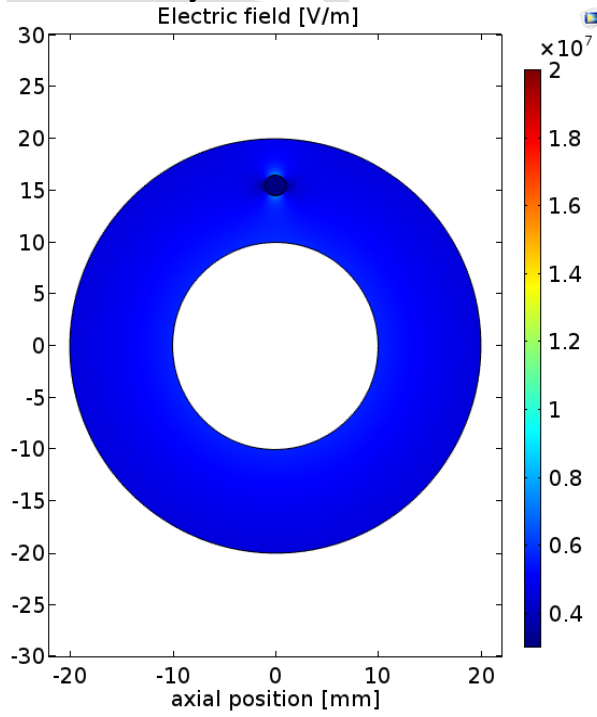

(b)

$\mathrm{C}$ cavity field $=4.7 \cdot 10^{6} \mathrm{~V} / \mathrm{m}$

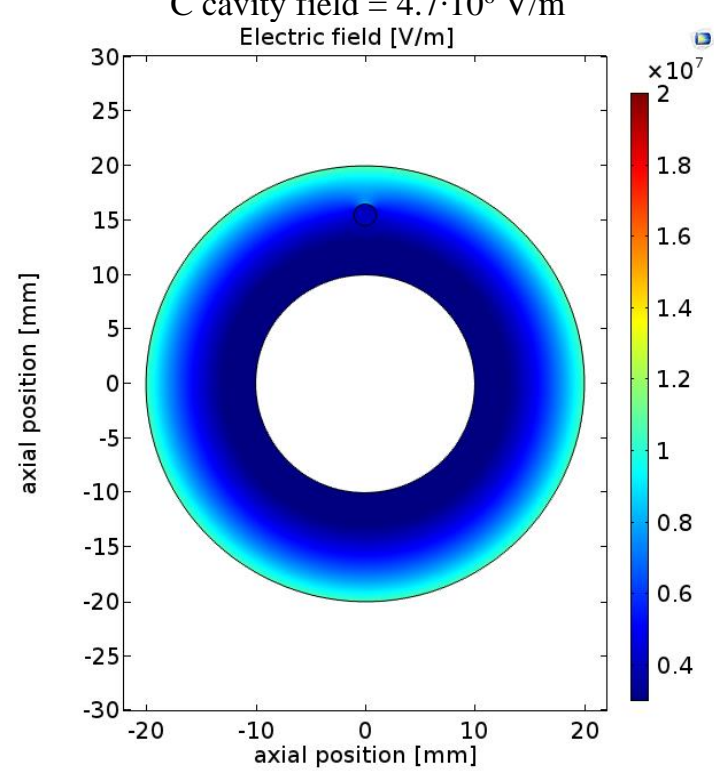

(c)

Fig. 2. Electric field simulation in a MV XLPE cable with a spherical internal defect filled with air under (a) AC and (b, c) DC power supply. 
Conductor (Al) electrical conductivity: $3.50 \cdot 10^{7} \mathrm{~S} / \mathrm{m}$, reference polymer electrical conductivity: $\sigma_{0}=5 \cdot 10^{-17} \mathrm{~S} / \mathrm{m}$, temperature coefficient $\alpha=0.05$, polymer relative permittivity: $\varepsilon_{r}=2.3$, cavity gas electrical conductivity:
$1 \cdot 10^{-15} \mathrm{~S} / \mathrm{m}$. Figure 2 (b) is obtained when the inner conductor is at $45^{\circ} \mathrm{C}$, while Fig. 2 (c) when the inner conductor is at $90^{\circ} \mathrm{C}$. Voltage $50 \mathrm{kV}$ peak. Outer conductor temperature fixed at $35^{\circ} \mathrm{C}$.

TABLE 1. VALUES OF RELATIVE PERMITTIVITY AND CONDUCTIVITY AT $60^{\circ} \mathrm{C}$ FOR THE THREE MATERIALS CONSIDERED IN THIS PAPER, AS WELL AS ESTIMATED VALUES OF TIME CONSTANT ACCORDING TO EQNS. (1) AND (4).

\begin{tabular}{|c|c|c|c|c|c|}
\hline Material & $\begin{array}{c}\text { Relative } \\
\text { permittivity }\end{array}$ & Conductivity & $\begin{array}{c}\text { Time constant } \boldsymbol{\tau}_{\mathbf{1}} \\
\text { (Equation (1)) }\end{array}$ & $\begin{array}{c}\text { Time constant } \boldsymbol{\tau}_{\mathbf{2}} \\
\text { (Equations (3),(4)) }\end{array}$ & $\begin{array}{c}\text { Time to reach } \\
\text { steady state }\end{array}$ \\
\hline $\mathrm{XLPE}$ & 2.3 & $1.4 \cdot 10^{-15} \mathrm{~S} / \mathrm{m}$ & $1.45 \cdot 10^{4} \mathrm{~s}$ & $0.3 \cdot 10^{4} \mathrm{~s}$ \\
\hline $\mathrm{PP}$ & 2.4 & $1 \cdot 10^{-14} \mathrm{~S} / \mathrm{m}$ & $2.1 \cdot 10^{3} \mathrm{~s}$ & $0.9 \cdot 10^{3} \mathrm{~s}$ & $4.5 \cdot 10^{4} \mathrm{~s}$ \\
\hline $\mathrm{PP}+\mathrm{SiO}_{2}$ & 2.6 & $6 \cdot 10^{-16} \mathrm{~S} / \mathrm{m}$ & $3.8 \cdot 10^{4} \mathrm{~s}$ & $0.6 \cdot 10^{4} \mathrm{~s}$ & $2.6 \cdot 10^{3} \mathrm{~s}$ \\
\hline
\end{tabular}

Focusing on the electric field transient, it can be then speculated that even if an insulation system is designed to operate below PDIV $\mathrm{DC}_{\mathrm{DC}}$, in the presence of insulation defects, as cavities filed by air, it is very likely that at each energization, when the electrical apparatus (e.g. a cable) is unloaded, PD will incept. Being the repetition rate very large compared to DC, due to the time variation of electric field in a defect, this can contribute non-negligibly to insulation premature failure. It becomes then of utmost importance to estimate the value of $\tau$, since the longer $\tau$, the higher the potential damage caused by $\mathrm{PD}$ and temporary field enhancement. A way to estimate the quantities in eq. (1) is suggested in [12], that is, resorting to measurements of the charging current for the insulating material used in the apparatus under consideration, close to the design field and in the operating temperature range. In this way, values of $\tau(E, T)$ can be estimated and used as the reference time to estimate the electric field transient duration.

\section{EXPERIMENTAL RESULTS}

Measurements of polarization current were performed on flat, extruded specimens provided by partners of the European project GRIDABLE. One of the tested materials was manufactured adding properly functionalized silica, $\mathrm{SiO}_{2}$, to PP compounds as described in [1-5], having permittivity and conductivity at $60^{\circ} \mathrm{C}$ as reported in Table 1 .

Conductivity was measured by an electrometer, connected to a high-impedance input module, and a guardedelectrode sample holder, at fields near to those used for the PD measurements and $60^{\circ} \mathrm{C}$ (see Figure 3). A continuous monitoring of the detected conductivity was recorded, until a stable reading was reached. Permittivity and dissipation factors were measured at a fixed frequency of $50 \mathrm{~Hz}$ by a dielectric analyzer at $60^{\circ} \mathrm{C}$.

Considering the obtained values of steady-state conductivity, $\sigma_{T}$, and of relative permittivity $\varepsilon_{r}$, a first estimate of the time constant, i.e. $\tau_{l}$, is provided in Table 1.

The values of time constants were also estimated also through an interpolating model, according to the equivalent circuit of Fig.6, [12], that is:

$$
I(t)=I_{0}+\sum_{i} I_{i} \exp \left(-\frac{t}{\tau_{i}}\right)
$$

where $I(t)$ is the time trend of the detected polarization current, $I_{0}$ is the steady state conduction current, $I_{i}$ and $\tau_{i}$ are constants associated to the displacement current and activation frequencies of different polarization mechanisms for each specific material. Experimental values of the time $t_{s s}$ required to reach a steady state condition of the depolarization current (where $I(\tau) \sim I_{0}=0$, Figure 4) are reported in Table 1. This time is related mostly to the slowest polarization mechanism, i.e. interfacial polarization. Once this stabilization time $t_{s s}$ is known, an estimate of the time constant reported in Table 1 as $\tau_{2}$ can be calculated, since it is commonly accepted that the time required for an exponential transient to fully develop is equivalent to about 5 time constants or:

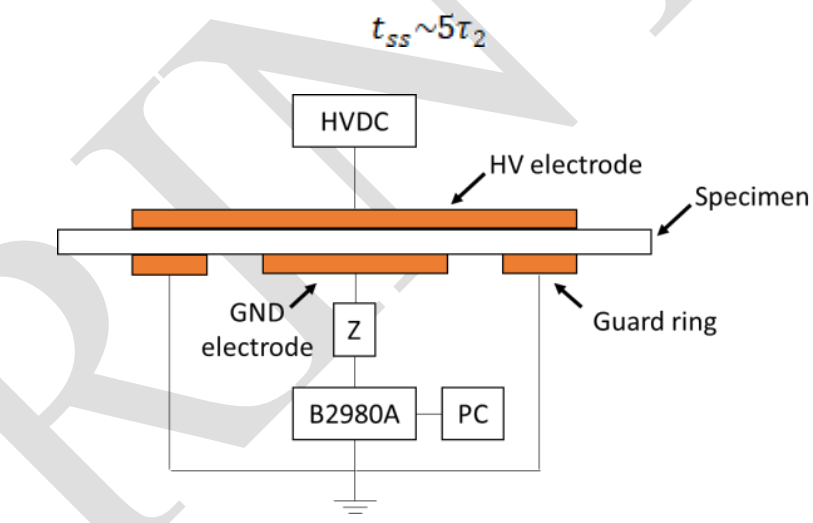

Fig. 3. Scheme of the conduction current measurement cell.

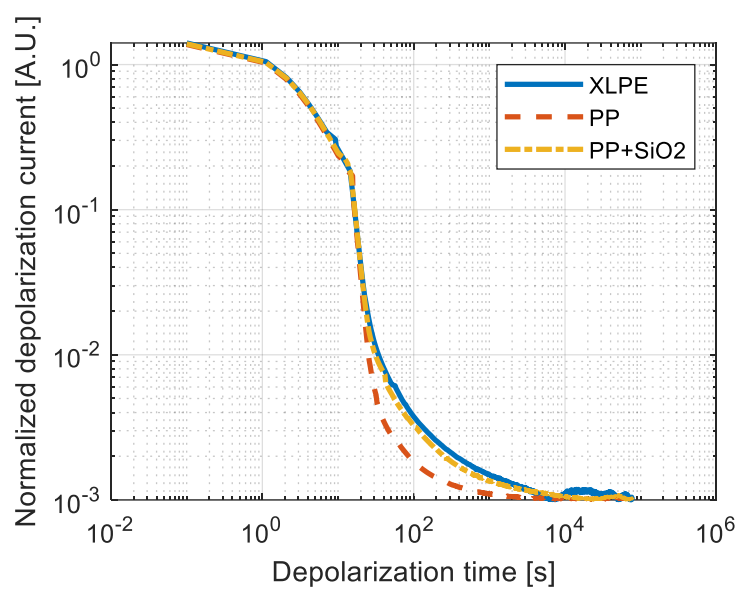

Fig. 4. Example of normalized depolarization currents measured for each material of Table 1 


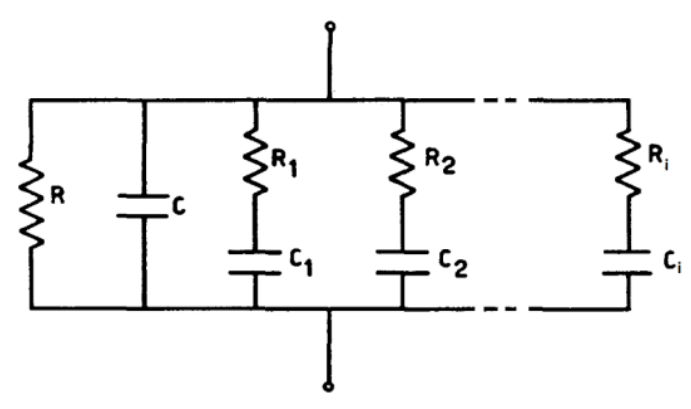

Fig. 5. Equivalent circuit of a real dielectric with a different R-C branch for each polarization mechanism of the material. The time constants, $R_{i} C_{i}=$ $\tau_{\mathrm{i}}$, are related to the inverse of relaxation frequencies for each mechanism.

\section{DISCUSSION}

The fitting of the values of electric field time constant, $\tau_{2}$, calculated by eq. (4), and those estimated by the ratio $\tau_{1}=\varepsilon_{\mathrm{r}} / \sigma_{T}$ from the permittivity and conductivity measurements are different, but the extent of the deviation can be negligible, considering measurement errors, or noticeable depending on material polarization characteristics. The equivalent permittivity $\varepsilon_{r}$ in eq. (1), obtained from conventional bridge measurement devices, does not account for the global polarization processes influencing the charging current behavior, and the numerical value of conductivity is obtained only once steady state conditions are met in the dielectric. This may make eq. (1), for some materials affected by significant low-frequency polarization processes and having large field and temperature dependence of conductivity, less robust than that based on depolarization current. For the tested materials, however, $\tau_{l}$ very is close to the calculated full duration of the transient, $t_{s s}$, (see Table 1). Another way to estimate the duration of transients in dielectrics can be done indirectly resorting to the time for a specimen having an internal cavity to reach a steady state value of the repetition rate of partial discharges. For this purpose, PD measurements were performed applying a voltage step from 0 to $10 \mathrm{kV}$ of amplitude with a $1 \mathrm{kV} / \mathrm{s}$ slope on three-layer specimens of the three investigated materials, having the central layer punctured in order to produce a cavity with a $3 \mathrm{~mm}$ diameter and $0.4 \mathrm{~mm}$ thickness. Detection and monitoring of PD was performed with an oscilloscope, (1 GHz, $200 \mathrm{MS} / \mathrm{s}$ sampling rate) endowed with dedicated acquisition and processing software, using large bandwidth HFCT. Polypropylene blends were mixed with a Kraussmaffei Berstorff ZE 25/49D twin screw extruder, and extrusion of samples was done by Brabender single screw extruder in a soft wall clean room. The nanoparticles were kept in an oven for 1.5 hours at $70^{\circ} \mathrm{C}$ and then additionally for $30 \mathrm{~min}$ at $70^{\circ} \mathrm{C}$ in vacuum to remove any potential moisture.

Temperature was fixed at an isothermal condition of $60^{\circ} \mathrm{C}$. The supply voltage was increased at a rate of $1 \mathrm{kV} / \mathrm{mm}$ up to a constant value of $10 \mathrm{kV}$. Figure 6 shows the PD repetition rate (rr) trend over time for the two polypropylene materials of Table 1 . The first thing to address is that PD rr is much higher in the material with lower conductivity (PP), both during the initial transient and at steady state. This can be explained by the theory of cavity charging after a PD, which takes less time if conductivity is smaller [14]. It can be also seen that the rr decreases going from the beginning of the energization transient to the steady state conditions, stabilizing after a time period comparable to the one experimentally found from depolarization currents, and indeed similar to the time predicted by eq. (1) $\left(\tau_{l}\right)$.

\section{CONCLUSIONS}

In conclusion, this paper highlights that since the energization of DC apparatus and, when needed, change of polarity, causes modifications in the electric field distribution, which is driven by permittivity during the initial phases of the transient (when field varies with time) and gradually drifts towards a conductivity-driven profile in steady-state, insulation defects will have potentially the capability to cause highly energetic degradation phenomena in insulations during each energization transient (such as PD or thermal instability). The duration of those transients is mostly related to conductivity, hence it will change depending on load conditions, supply voltage variation and location of defects.

This makes insulation system design much more complex under $\mathrm{DC}$ rather than $\mathrm{AC}$, because it is not only a matter to choose the design field, but also to ascertain that significant accelerated aging does not occur locally during supplyvoltage time variation.

Based on the above results, we have contrasting insulation design indications. On one hand, short transient time to establish the DC field would be appropriate (to reduce PD extent and related degradation, Fig.1 ), which can

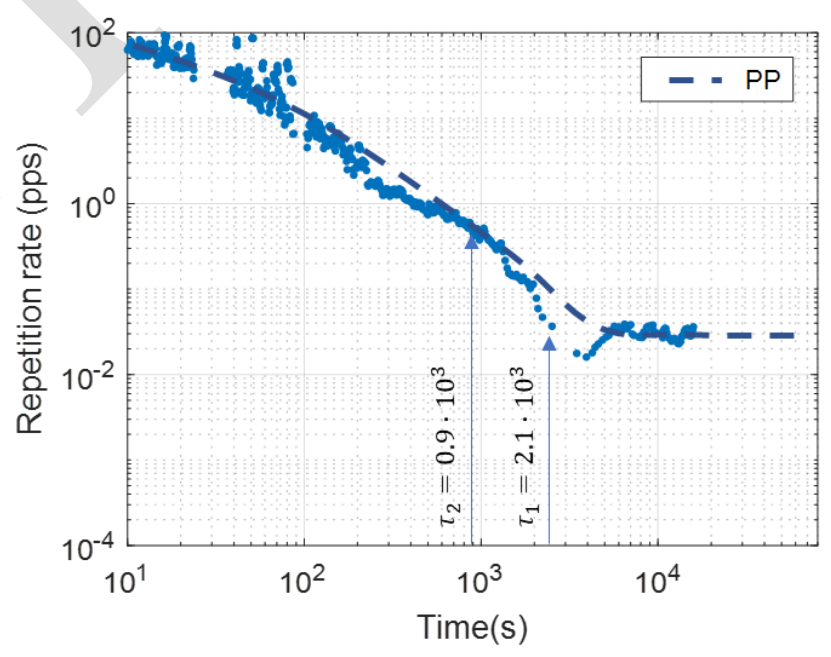




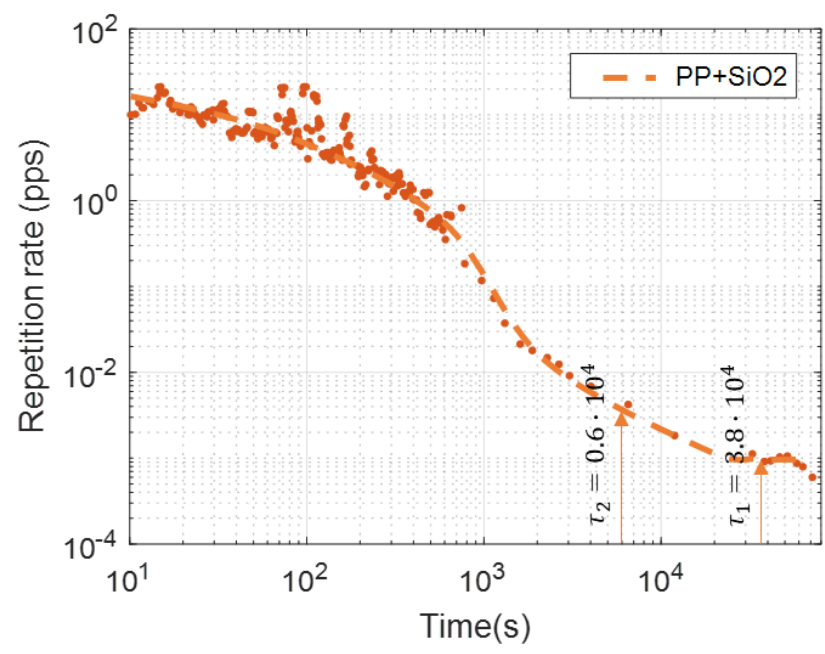

Fig. 6. Comparison of the values of time constant estimates $\tau_{l}$ and $\tau_{2}$ (Table 1) with the PD repetition rate behavior for materials based on PP. Measurements performed at $60^{\circ} \mathrm{C}$.

be accomplished by fast depolarization current, Fig. 4. On the other hand, high partial discharge inception voltage in DC, PDIV $V_{\text {DC }}$, would be needed, which could be achieved by materials having small conductivity and dependence of conductivity on temperature ( $\alpha$, eq. (2)) [6]. Small conductivity and fast relaxation processes seem to be the winning combination on which insulating material choice should be based.

Hence, charging current measurements are very helpful to estimate the time duration of the field transient, for an insulating material, thus allowing potentially evaluation of accelerated aging caused by each energization or voltage polarity inversion in the presence of defects having PDIV $_{A C}$ lower than operating voltage.

It is noteworthy that an important aspect not considered in this approach is the accumulation of space charge [15]. If space charges are trapped in insulation, at the defect (cavity) interface, they can influence the field in the cavity at the beginning of energization, thus time constant $\tau$, as well as the value of steady state PDIV $\mathrm{DC}_{\mathrm{DC}}$. This deserves certainly further investigation.

\section{ACKNOWLEDGMENT}

This project has received funding from the European Union's Horizon 2020 research and innovation program under grant agreement No 720858.

\section{REFERENCES}

[1] G. C. Montanari, P. Seri, M. Ritamäki, K. Lahti, I. Rytöluoto and M. Paajanen, "Performance of nanoparticles in the electrical behavior of DC capacitor films," 2018 12th International Conference on the Properties and Applications of Dielectric Materials (ICPADM), Xi'an, 2018, pp. 41-44.

[2] Rytöluoto, M. Ritamäki, and K. Lahti, "Short-Term Dielectric Performance Assessment of BOPP Capacitor Films: A Baseline Study," in 12th International Conference on the Properties and Applications of Dielectric Materials (ICPADM), 2018.

[3] I. Rytöluoto et al., "Compounding, Structure and Dielectric Properties of Silica-BOPP Nanocomposite Films," 2018 IEEE 2nd International Conference on Dielectrics (ICD), Budapest, 2018, pp. 1-4.

[4] G. C. Montanari et al., "Potentiality of nanofilled thermoplastic insulation for DC cables and capacitors," 2018 IEEE 2nd International Conference on Dielectrics (ICD), Budapest, 2018, pp. 14.

[5] Gian Carlo Montanari, Paolo Seri, Mikko Karttunen, Mika Paajanen, Kari Lahti, Ilkka Rytöluoto, "Investigation of Nanocomposite Polypropylene for DC Capacitors: A Feasibility Study," IEEJ Transactions on Fundamentals and Materials, 2019, Volume 139, Issue 2, Pages 105-112, Released February 01, 2019

[6] G.C. Montanari, R. Hebner, P. Seri, H. Naderiallaf, "Partial discharge inception voltage and magnitude in polymeric cables under AC and DC voltage supply," Jicable, pp. 1-4, Varsailles, France, July 2019

[7] P. Seri, L. Cirioni, H. Naderiallaf, G.C. Montanari, R. Hebner, A. Gattozzi, X. Feng, "Partial Discharge Inception Voltage in DC insulation systems: a comparison with AC voltage supply," IEEE Electr. Insul. Conf. (EIC), pp. 1-4, Calgary, Canada, June 2019.

[8] F. Kreuger, Industrial High DC Voltage, Delft University press, 1995.

[9] IEEE P 400.5, Guide for Field Testing of Shielded DC Power Cable Systems Using High Voltage Direct Current (HVDC), preliminary draft, 2019.

[10] G. Mazzanti, "Including the Calculation of Transient Electric Field in the Life Estimation of HVDC Cables Subjected to Load Cycles," IEEE Electr. Insul. Mag., Vol. 34, No. 3, pp.27-37, 2018.

[11] G.C. Montanari, P. Seri, R. Ghosh, "Partial discharge measurements of DC insulation systems: the influence of the energization transient," 2019 IEEE Conf. on Electr. Insul. Dielectr. Phen. (CEIDP), Richland, WA, USA, 2019, to be published.

[12] E. Occhini and G. Maschio, "Electrical Characteristics of OilImpregnated Paper as Insulation for HV DC Cables, " IEEE Transactions on Power Apparatus and Systems, vol. PAS-86, no. 3, pp. 312-326, March 1967.

[13] L. Niemeyer, "A generalized approach to partial discharge modeling," IEEE Trans. Dielectr. Electr. Insul., Vol. 2, No. 4, pp. 510-528, 1995.

[14] P. H. F. Morshuis, J. J. Smit, "Partial discharges at DC voltage: their mechanism, detection and analysis," IEEE Trans. Dielectr. Electr. Insul., Vol. 12, pp. 328-340, 2005.

[15] G.C. Montanari, "A contribution to unravel the mysteries of electrical aging under DC electrical stress: where we are and where we need to go,” IEEE Inter. Conf. Dielectr. (ICD), pp. 1-11, Budapest, Hungary, June 2018.

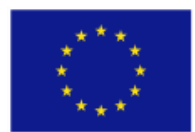

